\title{
Cardioversión eléctrica eficaz en posición prona en un paciente intubado con COVID-19
}

\author{
Alba Fernández Fernández-de Quincoces*, Nerea Azpiazu-Landa, María San Juan-González, \\ Silvia Valdivieso-Fernández y Sorkunde Telletxea-Benguria \\ Servicio de Anestesiología y Reanimación, Hospital de Galdakao-Usansolo, Vizcaya, España
}

\section{Resumen}

\begin{abstract}
Antecedentes: Se presenta el caso de un varón de 74 años que ingresa en la Unidad de Reanimación por neumonía bilateral por COVID-19. Intubado y en decúbito prono, experimentó varios episodios de taquiarritmia supraventricular, que exigieron cardioversión eléctrica efectiva. Métodos: Descripción de un caso clínico y revisión de las publicaciones médicas. Discusión: En esta nueva situación de pandemia por COVID-19 es preciso estar preparados para enfrentar situaciones diferentes a las habituales, con muchos de los pacientes colocados en decúbito prono, además de soportar la carga física y las limitaciones que implica vestir el equipo de protección individual. Aunque se desconoce la eficacia de algunas maniobras, como RCP o cardioversión en posición prona, algunos estudios comunican resultados exitosos. Conclusiones: A pesar de que no existe mucha bibliografía sobre la RCP y cardioversión en posición prona, en este caso fueron efectivas y deben ser una opción a considerar.
\end{abstract}

Palabras clave: Cardioversión eléctrica. Posición prona. COVID-19.

\section{Effective electrical cardioversion in a prone intubated COVID-19 patient}

\begin{abstract}
Background: We present the case of a 74-year-old man who was admitted in the Intensive Care Unit due to a bilateral pneumonia caused by COVID-19. While he was intubated and in prone position, he presented several episodes of supraventricular tachyarrythmia, requiring electrical cardioversion, which was effective. Methods: Description of a clinical case and review of the literature. Discussion: In this COVID-19 pandemic situation, we must be prepared to face new different scenarios, with many of the patients in the prone position, in addition to supporting the physical load and the limitations of wearing the personal protective equipment. Although the efficacy of performing some maneuvers such as CPR or cardioversion in the prone position is unknown, there are some studies that report successful results. Conclusions: Although there is not much bibliography about CPR and cardioversion in prone position, in our case it was effective, and we believe that it should be an option to consider.
\end{abstract}

Key words: Electrical cardioversion. Prone. COVID-19.

\section{Correspondencia:}

*Alba Fernández Fernández-de Quincoces

Fecha de recepción: 20-09-2020

Fecha de aceptación: 13-05-2021

DOI: 10.24875/RAA.20000005
Disponible en internet: 19-07-2021

Rev Argent Anestesiol. 2020;78:9-11 www.revistaargentinaanestesiologia.com 


\section{Introducción}

Durante la pandemia del COVID-19, el número de pacientes ingresados en unidades de cuidados críticos por insuficiencia respiratoria ha aumentado de manera significativa; muchos de ellos se han encontrado en estado de hipoxemia grave y se han beneficiado de maniobras como la posición prona para mejorar la oxigenación. La hipoxemia y algunos otros trastornos, entre ellos la acidosis, las alteraciones electrolíticas e incluso los efectos adversos de fármacos como la hidroxicloroquina, pueden favorecer el desarrollo de arritmias o el colapso circulatorio que exija cardioversión. Debido a que muchos de estos pacientes pueden encontrarse en posición prona, puede ser necesario realizar estas maniobras en esa posición.

\section{Caso clínico}

Varón de 74 años, alérgico a los betalactámicos y con hipercolesterolemia en tratamiento con pitavastatina, que ingresa en la Unidad de Reanimación por neumonía bilateral por COVID-19 y con necesidad de sedación y analgesia, intubación orotraqueal y conexión a ventilación mecánica.

Al cuarto día de ingreso, ante empeoramiento clínico y gasométrico, se decide colocarlo en decúbito prono, tras lo cual se observa taquiarritmia hasta de $200 \mathrm{lpm}$, con discreta hipotensión, que remite a $115 \mathrm{lpm}$ luego de administrar amiodarona (300 mg). Se comienza también perfusión de noradrenalina a 0.2-0.25 $\mathrm{gg} / \mathrm{kg} / \mathrm{min}$.

Asimismo, aparecen febrícula mantenida hasta la fiebre franca $>39{ }^{\circ} \mathrm{C}$ y un repunte de los parámetros sépticos analíticos. Se extraen cultivos y se indican de forma empírica tigeciclina, linezolid y amikacina.

La evolución es tórpida, con desarrollo de choque séptico. El quinto día de ingreso aumentan los requerimientos de noradrenalina hasta $1 \mu \mathrm{g} / \mathrm{kg} / \mathrm{min}$ sin obtener respuesta hemodinámica. Persiste la insuficiencia renal pese a la perfusión de furosemida, que se convierte en anúrica tan solo 24 horas después. Desde el punto de vista respiratorio, se establece empeoramiento gasométrico con déficit en la oxigenación y escasa reacción a los diferentes cambios posturales.

En el monitor se registran secuencias de taquiarritmia autolimitadas hasta de $170 \mathrm{lpm}$ al parecer en ACFA, con hipotensión adjunta, que se alternan con taquicardia supraventricular paroxística, con la misma frecuencia y estabilidad hemodinámica. Se administra diltiazem (12.5 mg IV) con respuesta parcial.
A lo largo de la mañana, con el paciente en posición prona, aparece una secuencia de taquicardia supraventricular a 175 Ipm con gran repercusión hemodinámica, por lo que se decide realizar una cardioversión eléctrica sincronizada. En esa situación no se dispone ni de tiempo ni de personal para colocar al paciente en posición supina, por lo que se colocan las palas en posición posterolateral (escápula derecha y línea axilar izquierda), con aplicación de una descarga bifásica de $100 \mathrm{~J}$; se obtiene tras el primer choque ritmo sinusal a $45 \mathrm{lpm}$ e hipotensión que exige dosis altas de noradrenalina $(2 \mu \mathrm{g} / \mathrm{kg} / \mathrm{min})$ y adrenalina $(0.02-0.03 \mu \mathrm{g} / \mathrm{kg} / \mathrm{min})$, y se consigue una FC de $124 \mathrm{lpm}$ y TA de $119 / 47 \mathrm{mmHg}$. Al cabo de unas horas es posible retirar la PC de adrenalina.

Cuarenta y ocho horas después del inicio del empeoramiento, la situación se vuelve catastrófica con evolución al choque séptico y crecimiento de neumococos en hemocultivos, inestabilidad hemodinámica que no responde a dosis máximas de vasopresores y anuria. Se registra agravación respiratoria y gasométrica con acidosis respiratoria pese a la posición prona, $\mathrm{FiO}_{2}$ máxima y cambios posturales ineficaces.

Debido a la inutilidad de las medidas aplicadas, se decide efectuar una limitación del esfuerzo terapéutico con desenlace letal el sexto día luego de ingresar a reanimación.

\section{Discusión}

Como se ha mencionado ya, en esta pandemia COVID-19 se han enfrentado nuevos escenarios y se ha atendido a pacientes muy graves con una infección altamente contagiosa, además de soportar la carga física que supone vestir el equipo de protección individual y las limitaciones que implica llevar a cabo ciertas maniobras'.

Los desafíos relacionados con el cuidado pulmonar de estos pacientes han llevado a iniciar de manera constante protocolos de ventilación mecánica en posición prona, lo que supone un reto al efectuar maniobras emergentes, por ejemplo la cardioversión eléctrica sincronizada o la RCP 2,3 .

Existe bibliografía acerca de la RCP en posición prona, en intervenciones neuroquirúrgicas o raquídeas, y aunque se desconoce su efectividad en esta posición, existen algunos estudios e informes de casos que comunican resultados exitosos ${ }^{4}$.

Los pacientes COVID-19 colocados en posición prona podrían ser otra de las indicaciones para este procedimiento. Las razones de esta indicación son a) 
intentar reducir al mínimo la exposición del personal, ya que se trata de una afección de alta contagiosidad; b) disminuir el tiempo de actuación, dado que la probabilidad de éxito de la descarga depende de la rapidez con la que se aplique y toma cinco minutos llevar al paciente a la posición supina; y c) abatir el riesgo de pérdida de vías o extubación accidental, que además de acarrear consecuencias desastrosas para el paciente aumentaría aún más el riesgo de contagio por la generación de aerosoles. Además, esto garantiza poder actuar de inmediato, ya que es posible que no se cuente con la ayuda necesaria (al menos cinco personas para colocar en posición supina al enfermo) en el momento requerido ${ }^{5,6}$.

En caso de realizar RCP en posición prona se recomienda colocar las manos sobre la línea media a la altura de las escápulas, durante un ciclo de reanimación de dos minutos. En otros contextos, si no es efectiva, se recomienda girar al paciente. En este caso es preciso determinar si la posición supina tiene utilidad para obtener mayor probabilidad de éxito, sin soslayar los riesgos descritos con anterioridad ${ }^{4}$.

Para realizar una cardioversión o desfibrilación se colocan los electrodos de extremo en el quinto espacio intercostal de la línea axilar anterior izquierda y los electrodos del esternón en la región escapular derecha, con máxima energía posible. Esta recomendación tiene un grado de evidencia muy bajo y su único sustento es el de evitar al máximo la exposición del personal de salud. Los electrodos también pueden aplicarse en posición anteroposterior (uno a la izquierda del borde esternal inferior y otro posteriormente) o de forma bilateral sobre ambas líneas axilares medias ${ }^{7}$. Se recomienda la desfibrilación inmediata, en tres dosis de forma secuencial, sin practicar compresiones torácicas.

Algunas de las medidas para conseguir una cardioversión o desfibrilación más eficaces son la previsión de posibles episodios de arritmias, para lo cual se dejan colocadas los electrodos cuando el paciente se encuentra en decúbito supino, y se intenta administrar la descarga en la espiración con la mínima presión positiva al final de la espiración (PEEP), ya que aumenta la impedancia transtorácica.

Como conclusión, en esta nueva situación de pandemia por COVID-19 es necesario estar preparados para enfrentar situaciones diferentes a las habituales. A pesar de que no existe mucha bibliografía sobre la
RCP y cardioversión en posición prona, en este caso fue efectiva y debe ser una opción a tener en cuenta.

Este paciente mostró de modo inicial secuencias de taquiarritmia una vez colocado en posición prona, por lo que no fue posible adelantarse a colocar los electrodos antes de adoptar esta posición; empero, dado que había sido efectiva la descarga con electrodos, es de suponer que esta efectividad tendría mayores garantías en caso de colocar los electrodos al reducir al mínimo el contacto directo del reanimador con el paciente.

\section{Financiamiento}

La presente investigación no ha recibido ninguna beca específica de agencias de los sectores públicos, comercial o sin ánimo de lucro.

\section{Conflicto de intereses}

Las autoras declaran no tener conflicto de intereses alguno.

\section{Responsabilidades éticas}

Protección de personas y animales. Los autores declaran que para esta investigación no se han realizado experimentos en seres humanos ni en animales.

Confidencialidad de los datos. Los autores declaran que han seguido los protocolos de su centro de trabajo sobre la publicación de datos de pacientes.

Derecho a la privacidad y consentimiento informado. Los autores declaran que han obtenido el consentimiento informado del paciente referido en el artículo.

\section{Bibliografía}

1. Tran K, Cimon K, Severn M, Pessoa-Silva CL. Aerosol generating procedures and risk of transmission of acute respiratory infections to healthcare workers: a systematic review. PloS one. 2012;7(4):e35797.

2. Cattell E, Saravanan P, Chay S, Lawler PG. The defibrillator back paddle: use for treatment of arrhythmias during prone position ventilation. Anaesthesia 2000:55:491.

3. Fikkers BG, Bello JL, van Dijk APJ. Electric cardioversion of atrial flutter in a critically ill patient in the prone position. 2001;27(2):449.

4. Aguirre MM. Paro cardiorrespiratorio (PCR) y reanimación cardiopulmonar (RCP) en un nuevo escenario: COVID19. Números. 2020;(1):2019. Disponible en: https://doi.org/10.25237/revchilanestv49n03.017

5. Mędrzycka-Dąbrowska W, Lewandowska K, Ślęzak D. Prone ventilation of critically ill adults with COVID-19: how to perform CPR in cardiac arrest? Critical Care. 2020;24(1):1-2.

6. Bamford P, Denmade C, Newmarch C. Guidance for prone positioning in adult critical care. Prepared on behalf of the Intensive Care Society and Faculty of Intensive Care Medicine. 2019.

7. Miranda CC, Newton MC. Successful defibrillation in the prone position. British Journal of Anaesthesia. 2001;87(6):937-938. 